\title{
Personalidade e psicopatia: implicações diagnósticas na infância e adolescência
}

\author{
Tárcia Rita Davoglio \\ Gabriel José Chittó Gauer \\ João Vitor Haeberle Jaeger \\ Marina Davoglio Tolotti \\ Pontifícia Universidade Católica do Rio Grande do Sul
}

\begin{abstract}
Resumo
Este artigo de revisão de literatura examinou o construto da psicopatia associado ao desenvolvimento da personalidade em crianças e adolescentes, privilegiando as questões diagnósticas incipientes. Observou-se que a busca de uma terminologia mais apropriada para descrever as manifestações desviantes precoces, a construção e utilização de instrumentos de avaliação dirigidos à psicopatia em jovens, bem como a estabilidade dos sintomas ao longo do desenvolvimento, têm sido preocupações recorrentes nas pesquisas atuais. Podese afirmar que a presença de traços de psicopatia na infância e adolescência ainda suscita questionamentos, demandando por estudos empíricos que explorem aspectos evolutivos e a etiologia multifatorial do construto, preferencialmente, dentro da concepção geral dos transtornos de personalidade.
\end{abstract}

Palavras-chave: personalidade; infância e adolescência; comportamentos antissociais; traços de psicopatia.

\begin{abstract}
Personality and psychopathy: diagnostic implications in childhood and adolescence. This literature review article examined the psychopathy construct associated to the development of personality in children and adolescents focusing on the incipient diagnostic issues. It was observed that the search for a more appropriate terminology for describing the early deviant manifestations, the building and using of instruments of evaluation directed to psychopathy in youngsters as well as the stability of the symptoms during growth have been recurrent issues in current researches. It can be affirmed that the presence of psychopathy traits in childhood and adolescence still raises doubts, requiring empirical studies which explore the evolutive aspects and the multifactorial etiology, preferentially within the general conception of personality disorders.
\end{abstract}

Keywords: personality; childhood and adolescence; anti-social behavior; psychopathy traits.

A s pesquisas contemporâneas têm apontado nas experiências infantis a presença de fatores de risco elevado para o desenvolvimento de quadros psicopatológicos. Uma revisão sobre o desenvolvimento de transtornos de personalidade (Johnson et al., 2005) relacionou diversos estudos confirmando que crianças e jovens submetidos a experiências traumáticas (tais como abuso físico/psicológico, negligência, doença mental parental, punição excessiva e agressiva) seriam, potencialmente, mais vulneráveis à presença de traços ou sintomas de transtornos de personalidade. Estes, depois de cristalizados, associam-se fortemente à violência, abuso de drogas, tentativas de suicídio, comportamentos destrutivos e criminosos, institucionalização, prejuízo global no rendimento e desorganização familiar (Ruegg \& Frances, 1995).

Segundo o Manual Diagnóstico e Estatístico de Transtornos Mentais (DSM-VI-R; Associação Psiquiátrica Americana [APA], 2002), os traços de personalidade são expressos por padrões persistentes de perceber, relacionar-se com e pensar sobre si mesmo ou sobre o ambiente. Quando se mostram inflexíveis e desadaptativos para a cultura do sujeito, causando mal estar subjetivo ou prejuízo funcional importante, podem caracterizarse como um transtorno. Um transtorno de personalidade (TP), independente de sua tipologia específica, manifesta-se, então, em pelos menos duas áreas da vida, envolvendo a cognição, a afetividade, o funcionamento interpessoal ou controle dos impulsos, não sendo apenas resposta a estressores específicos. Ainda assim, o contato com a realidade mantém-se preservado e, quase sempre, esses traços não são percebidos pelo sujeito como estranhos ou indesejáveis, o qual avalia positivamente (portanto, de modo equivocado) o impacto dos mesmos sobre o ambiente (O’Connor \& Dyce, 2001).

As duas principais classificações nosológicas dos transtornos mentais em uso (APA, 2002; Organização Mundial da Saúde [OMS], 2007) não recomendam o diagnóstico nosográfico de TP 
antes dos 17 ou 18 anos. Porém, concordam que esses transtornos iniciam-se precocemente e tendem a durar quase toda a vida, sendo relativamente frequentes entre a população geral, com prevalência entre 10 a 13\% (Abrams \& Horowitz, 1996). Para clínicos e pesquisadores, são transtornos que não surgem de modo repentino, tendo precursores de suas características básicas presentes ainda na infância, consolidando-se e estabilizando-se posteriormente (O’Connor \& Dyce, 2001), levando à suposição de que é possível obter indícios diagnóstico, baseados em evidências substanciais, antes da idade adulta.

Então, sem desconsiderar as recomendações dos manuais, as evidências provindas da prática clínica e psicossocial acusam a existência, no mínimo, de traços muito expressivos de TP em idades precoces, trazendo à tona benefícios e riscos da utilização de nomenclaturas mais específicas. As atuais recomendações, assim, contribuem para deixar em aberto um desafiador debate, ainda sem consenso, dirigido às questões diagnósticas associadas ao momento evolutivo em que os TP podem de fato ser identificados. Esta polêmica é ainda mais específica diante do transtorno de personalidade antissocial (TPAS ${ }^{1}$; APA, 2002), pois a ele se associam implicações jurídicas e/ou sociais significativas (Davoglio \& Argimon, 2010).

No entanto, a própria evolução na abordagem clínica dos TP com adultos foi responsável pela intensificação das discussões sobre a adaptação do conceito para crianças e adolescentes, especialmente, no que se refere à psicopatia (Salekin \& Frick, 2005). A psicopatia consiste em um TP que envolve tanto características comportamentais desviantes, quanto aspectos afetivos e relacionais, cuja etiologia e desenvolvimento ainda são pouco conhecidos. A busca de uma terminologia mais apropriada (Achenbach, 1991; Wangby, Bergman, \& Magnusson, 1999), bem como o desenvolvimento de instrumentos de avaliação dirigidos à psicopatia em jovens (Forth, Kosson \& Hare, 2003; Lynam, 1997) têm sido preocupação recorrente nas pesquisas atuais com esta população.

Esta revisão de literatura, realizada por meio de pesquisa em bases de dados (Pubmed, PsycInfo e Medline) e em material bibliográfico relacionado, sem a intenção de esgotar o tema, objetivou examinar o construto da psicopatia associado ao desenvolvimento da personalidade em crianças e adolescentes. Foram privilegiadas as questões diagnósticas dos comportamentos desviantes precoces sugestivos de traços incipientes de psicopatia. A partir de descritores amplos, em língua inglesa e portuguesa, como personalidade antissocial, transtorno de conduta, psicopatia e delinquência, relacionados à infância e à adolescência, foram localizados um grande número de artigos e obras disponíveis sobre a temática (em geral, em língua inglesa) produzidos especialmente nas duas últimas décadas. Na essência, os materiais não apresentaram grande diversidade de informações, porém, ressaltam questões abordadas neste artigo, que ainda continuam pouco exploradas, especialmente em relação à utilidade do construto ou a melhor forma de conceituá-lo, nomeá-lo e mensurá-lo na infância e na adolescência. Além disso, apontam para a necessidade de replicação de estudos empíricos, cujo foco localiza-se nas questões envolvendo as bases etiológicas e a possibilidade efetiva de diagnosticar o construto precocemente, melhorando a sua evolução clínica.

\section{Infância, adolescência e juventude no contexto da violência}

Devido à evolução das características de alguns transtornos ao longo da infância e adolescência e pelo agravamento da criminalidade envolvendo jovens (UNICEF, 2009), nas últimas décadas, passou-se a enfatizar a relevância clínica e diagnóstica dos problemas comportamentais de crianças com menos de seis anos, como indicadores de possíveis dificuldades futuras (Campbell, 1995). Nesta perspectiva, os autores não desvalorizam o processo pelo qual cada fase evolutiva é relevante e peculiar nas suas manifestações e na formação da personalidade (Kernberg,Weiner, \& Bardenstein, 2003).

De acordo com Pilotti (1995), os conceitos de infância e criança são complementares e interdependentes. $\mathrm{O}$ sentido da palavra criança pressupõe a dinâmica do desenvolvimento individual, apontando para a dimensão mais psicológica; a palavra infância remete à dinâmica social, histórica e cultural em que esta criança efetivamente se insere. No Brasil, a criança é legalmente definida e apresentada pelo Estatuto da Criança e do Adolescente (ECA; Brasil, 1990) como a pessoa que possui idade entre 0 e 12 anos incompletos.

A adolescência, por sua vez, constituiu um processo que se inicia com o amadurecimento biológico, durante o qual se acelera também o desenvolvimento cognitivo e a estruturação da personalidade (Organización Panamericana de la Salud [OPS], 1985). Abrange as idades de 10 a 19 anos, divididas em pré-adolescência (dos 10 aos 14 anos) e adolescência propriamente dita (de 15 a 19 anos), representando "uma das formações culturais mais poderosas da nossa época" (Calligaris, 2000, p.9), considerando que, neste sentido, a adolescência se aproxima do conceito de infância. Já a juventude, dos 15 aos 24 anos, resume uma categoria essencialmente sociológica, indicativa do processo de preparação do indivíduo para assumir o papel adulto na sociedade, tanto no plano familiar quanto no profissional (OPS, 1985).

Partindo-se desses pressupostos, examinar a infância e a adolescência contextualizadas com a violência e a criminalidade presentes na sociedade deste século traz a tona questões paradoxais. Se por um lado, crianças e adolescentes são muitas vezes vítimas de abusos, maus tratos e experiências traumáticas, de outro, podem ser agentes de atos violentos, que praticam precocemente e que podem persistir ou se cristalizar ao longo do tempo. Nas suas mais diversas manifestações a violência é aqui entendida, segundo a definição de Michaud (1989), quando durante uma interação um ou vários sujeitos agem, direta ou indiretamente, causando danos a uma ou a mais pessoas em graus variáveis, podendo atingir tanto a integridade física ou moral quanto as posses e participações simbólicas e culturais.

Considera-se que na infância e na adolescência muitas manifestações de agressividade, impulsividade, ansiedade e, em algumas ocasiões, até mesmo os comportamentos delinquentes, que podem ou não envolver violência, são comuns (Achenbach, 1991) ou caracterizam apenas sintomas isolados e transitórios (Forth \& Burke, 1998). Quando persistentes, repetitivos ou violentos passam a ter significado psicopatológico, podendo 
evoluir (Forth \& Burke,1998; Loeber, Burke, Lahey, Winters, \& Zera, 2000; Veirmeiren, 2003) ou serem parte um estágio inicial (Patterson, Reid, \& Dishion,1992; Patterson, DeGarmo, \& Knutson, 2000) de transtornos só formalmente reconhecidos na idade adulta, como o TPAS (APA, 2002) ou a psicopatia (Hare, 2003) Por esse motivo, neste estudo, os comportamentos violentos, transgressores, antissociais e psicopatas não são, necessariamente, equivalentes.

\section{Classificação das manifestações desviantes precoces}

A literatura internacional atual tem utilizado uma nomenclatura específica para referir problemas comportamentais que não se configuram ainda, pela sua intensidade e frequência, como categorias nosológicas, porém, evidenciam a existência de problemas contrastantes entre si, observados em estudos empíricos. Os termos internalização e externalização foram, então, difundidos na tentativa de fazer distinção entre problemas que envolvem, respectivamente, conflitos inerentes ao self e aqueles que dizem respeito à interação com o ambiente (Wangby et al.,1999), antes que tenham efetivamente se consolidado em transtornos.

Pesquisadores que se dedicam às investigações empíricas do comportamento infantil envolvendo agressividade, desobediência, intolerância à frustração, oposicionismo, impaciência, fraco controle dos impulsos, fugas, roubos, entre outros, acreditam que essas manifestações podem ser englobadas na definição operacional de problemas de externalização (Achenbach, 1991; Lambert, Wahler, Andrade, \& Bickman, 2001), relacionando-as intimamente com aspectos antissociais (Fischer, Rolf, Hasazi, \& Cummings, 1984). As questões de internalização, por sua vez, ficariam relacionadas aos problemas depressivos, ansiosos, queixas somáticas e retraimento social. Essa terminologia, diferente da encontrada na nosologia clássica, tende a facilitar a descrição de problemas com crianças pequenas, especialmente, ao referir-se às manifestações de externalização, evitando o agravo subjacente à expressão antissocial ou psicopata.

Os problemas de externalização e o comportamento antissocial são apontados como os que apresentam maior prevalência na infância entre os meninos, maior estabilidade ao longo do tempo e maior probabilidade de evoluírem para quadros clínicos mais graves na adolescência e na vida adulta, se comparados aos problemas de internalização (Pettit, Bates, Dodge, \& Meece, 1999; Wangby et al., 1999). Há evidências empíricas (Keenan \& Shaw, 1998; Patterson et al., 2000) que sustentam a hipótese de que os problemas de externalização podem ser identificados precocemente, já em torno dos 18 meses, e o início precoce seria um dos indicadores de maior gravidade do quadro e de maior tendência a persistir ao longo da vida (American Academy of Child and Adolescent Psychiatry [AACAP], 1997). Contudo, considera-se válido enfatizar que a tendência aos comportamentos de externalização não constitui um diagnóstico em si e, por isso, pode ser encontrada em crianças que nunca venham a desenvolver transtornos específicos.

Por outro lado, quando persistentes, os problemas de externalização abrangem ou associam-se aos sintomas encontrados nos critérios diagnósticos para transtorno de conduta
(TC), transtorno desafiador opositivo, transtornos disruptivos e, em parte, para o transtorno de déficit de atenção e hiperatividade (TDAH) (APA, 2002). Segundo diversos estudos revistos por Pacheco, Alvarenga, Reppold, Piccinnini e Hutz (2005) esta relação é mais frequente quanto menor a idade da criança. A tendência permanente para apresentar comportamentos incômodos e perturbadores ao ambiente, o envolvimento em atividades perigosas e até mesmo ilegais são encontrados na essência destes transtornos, especialmente o de conduta. São comportamentos que se caracterizam por causar maior impacto aos demais do que ao próprio sujeito, o qual não apresenta sinais de empatia, sofrimento psíquico ou constrangimento com as próprias atitudes (Earls, 1994).

Os transtornos de conduta, então, tipicamente, se manifestam muito cedo, entre o início da infância e a puberdade e podem persistir até a idade adulta (Robins, 1991), com taxas de prevalência que variam de 6 a $16 \%$ para o sexo masculino (APA, 2002). Podem ser acompanhados por comorbidades, como o TDAH, comportamento agressivo, déficit intelectual, convulsões e comprometimento do sistema nervoso central (por consumo de álcool/drogas no período pré-natal, infecções, uso de medicamentos, traumas cranianos), além de antecedentes familiares positivos para hiperatividade e comportamento antissocial. Essas comorbidades tendem a ser mais frequentes quando o transtorno de conduta inicia-se antes dos 10 anos. É frequente também o TC apresentar-se associado ao baixo rendimento escolar e a dificuldades de relacionamento com colegas, resultando em limitações acadêmicas e sociais ao indivíduo (AACAP, 1997).

Essas manifestações comportamentais persistentes não são estáticas, tendendo a se modificar gradual e progressivamente no decorrer do desenvolvimento infantil. Assim, os comportamentos antissociais mais graves, tais como, brigas com armas, assaltos e arrombamentos, geralmente são antecedidos por comportamentos mais leves, como trapacear, gazear aula, furtar objetos de baixo valor e mentir (Rutter, 1992). Com o tempo, pode somar-se ainda o consumo abusivo de álcool e drogas (Loeber \& Dishion,1983) e, especialmente nas meninas, ocorrer o surgimento de quadros depressivos ou ansiosos (Rutter, 1992).

É possível que as características típicas desses comportamentos antissociais variem, também, de acordo com a idade em que se iniciam. Moffitt (1993), a partir deste pressuposto, distinguiu dois grupos de antissociais: um grupo com padrão de comportamento antissocial persistente ao longo da vida e outro limitado à adolescência. No primeiro grupo, os comportamentos antissociais iniciam-se na idade pré-escolar, com reações manifestas de oposicionismo e desafio, os quais ao evoluir resultam em sérios problemas de conduta na adolescência e na vida adulta. No segundo grupo, os comportamentos antissociais são circunscritos à juventude, estando fortemente apoiados na vantagem imediata e pelo grupo de pares, sendo que estes adolescentes são capazes de manifestar atitudes pró-sociais quando percebidas como recompensadoras.

Por outro lado, alguns pesquisadores (Farrington, 1995; Veirmeiren, 2003) defendem a ideia de que esses comportamentos antissociais que ocorrem na infância são protótipos de comportamentos delinquentes que poderão se manifestar só 
mais tarde, vindo a assumir, inclusive, a forma de transtorno de personalidade. É, justamente, devido a todas essas questões ainda não bem esclarecidas, somadas à intensidade e frequência de delitos e crimes envolvendo meninos e meninas nos dias atuais, que nota-se um crescente interesse teórico e aplicado no construto da psicopatia dirigido à infância e à adolescência. (Chandler \& Moran, 1990; Forth, Hart, \& Hare, 1990; Trevothan \& Walter, 1989).

Segundo Farrington (2005), a definição de psicopatia sintetizada e utilizada nas pesquisas atuais é derivada das contribuições teóricas originalmente propostas sobre o construto e embasada em estudos empíricos. Envolve três importantes dimensões, além dos comportamentos antissociais em si, assim caracterizadas:

1. Um estilo interpessoal enganador e arrogante, incluindo desinibição ou charme superficial, egocentrismo ou um senso grandioso de autoestima; mentira, trapaça, manipulação e enganação.

2. Experiência afetiva deficiente, com pouca capacidade de sentir remorso, culpa e empatia; uma consciência fraca, insensibilidade, afeto superficial e falha em aceitar responsabilidade pelas ações (utilizando-se de negação, desculpas, etc.).

3. Um estilo de comportamento impulsivo ou irresponsável, incluindo tédio, busca contínua por emoção, falta de metas em longo prazo, impulsividade, falha em pensar antes de agir e um estilo de vida parasita (tais como, dívidas, hábitos de trabalho insatisfatórios).

Nessa perspectiva, baseada nas ideias de Cleckley (1941/1988) e nos avanços de Hare (2003), pode-se inferir que a psicopatia sempre envolve comportamentos antissociais, porém, nem todos os comportamentos antissociais devem ser atribuídos à psicopatia. Segundo Morana (2004), a psicopatia, basicamente, pode ser compreendida sob dois aspectos: o descritivo e o psicodinâmico. Do ponto de vista descritivo, acompanhando a perspectiva nosográfica, constitui um transtorno de personalidade com consequências interpessoais e sociais, que se manifestam no comportamento do sujeito, levando-o a condutas antissociais, espoliativas e não empáticas. Do ponto de vista psicodinâmico, ou seja, intrapsíquico, observa-se alterações na estruturação da personalidade, em especial nas questões inerentes ao narcisismo (Davoglio, no prelo), levando o indivíduo a estabelecer uma relação distorcida e autocentrada com as pessoas, com os afetos e com as normais sociais.

\section{Evolução da pesquisa com psicopatia na infância e adolescência}

A possibilidade de identificar e tratar transtornos de personalidade nas populações jovens é ainda incipiente, embora, em 1940, Cleckley já tivesse reconhecido a psicopatia como um transtorno de personalidade que tem suas raízes estabelecidas na infância e adolescência. Dados históricos (Salekin \& Frick, 2005) apontam que na mesma década, Karpman tentou organizar formalmente alguns eventos de caráter científico para discutir a aplicabilidade do construto da psicopatia a essas amostras jovens. Salekin e Frick (2005) descreveram diversos trabalhos realizados por pesquisadores como McCord e McCord e Quay, nos anos 60. McCord e McCord observaram em seus estudos com jovens que a psicopatia assumia características distintas daquelas encontradas em outros TC, sendo que apenas $14 \%$ da amostra estudada apresentavam características semelhantes aos traços de psicopatia, demonstrando que não eram quadros idênticos. Assim, já nesse momento havia preocupações com a classificação psicopatológica na infância, levando outro pesquisador, Quay, a propor que os avanços terapêuticos estariam diretamente associados a uma melhor definição do que seria um "não psicopata", tentando com isto definir subtipos de delinquência juvenil. Embora a intenção deste último autor estivesse direcionada para a distinção entre o que é um comportamento antissocial típico da adolescência e o que vai muito além, sua proposta foi criticada por acabar gerando confusão conceitual, dificultando ainda mais as escassas pesquisas da época.

Passado mais de meio século desde os anos de 1940, essas questões ainda continuam pouco exploradas do ponto de vista teórico e empírico, especialmente no que concerne a alguns pontos, tais como: a definição clara do conceito de psicopatia, suas bases etiológicas, a real possibilidade de ser reconhecida precocemente e se, com isto, poderia apresentar melhor evolução (Salekin \& Frick, 2005). Somente a partir dos anos 90 houve uma retomada dos estudos com crianças e adolescentes em relação ao construto da psicopatia, parcialmente devido à evolução das pesquisas com amostras adultas, que se revelaram promissoras. Modelos teóricos enfatizando os déficits cognitivos e afetivos característicos da psicopatia (Cooke \& Michie, 2001) surgiram ao lado de instrumentos de avaliação como o Inventário de Psicopatia de Hare - Revisado (PCL-R; Hare, 2003/ Morana, 2004), difundindo a descrição da psicopatia como um transtorno mais complexo do que o TPAS.

Pesquisas que tentaram associar possíveis traços de psicopatia com diagnósticos nosográficos já consolidados para a infância e adolescência também fortaleceram a aplicabilidade do construto às populações jovens. Pesquisadores como Lynam (1997; 1998) fomentaram a hipótese de que alguns TC poderiam ser melhor descritos como traços incipientes de psicopatia. Com base nisso, estudos empíricos com crianças que apresentavam comorbidades de TC, distúrbios de atenção, hiperatividade e impulsividade permitiram identificá-las com padrões de comportamento antissociais mais graves e agressivos e com uma série de déficits neuropsicológicos (por exemplo, pobres respostas na modulação afetiva, incapacidade para postergar gratificações), bastante semelhantes aqueles reconhecidos na psicopatia adulta.

Tais pesquisas com crianças e adolescentes geraram, gradualmente, o desenvolvimento de instrumentos e métodos de avaliação confiáveis e específicos para estas faixas etárias. Além de medidas de autorrelato, como o Childhood Psychopathy Scale (CPS; Lynam, 1997) e de relato de informantes como o Antisocial Process Screening Device (APSD; Frick \& Hare, 2001), surgiram importantes escalas de avaliação como o Hare Psychopathy Checklist: Youth Version (PCL:YV; Forth et al., 2003), pontuado através de informações provindas de entrevistas com o adolescente e de dados institucionais. Este, o PCL:YV, nos estudos recentes tem apontado a presença de dimensões distintas do construto da psicopatia, envolvendo aspectos interpessoais, 
afetivos e comportamentais (Cooke \& Michie, 2001; Forth et al., 2003), além das transgressões legais e criminais típicas das personalidades antissociais.

As pesquisas empíricas sobre a psicopatia em jovens utilizando o PCL:YV já revelaram dados importantes. De acordo com uma revisão de literatura realizada recentemente (Vincent, Odgers, McCormick, \& Corrado, 2008) os estudos apontaram que: adolescentes que apresentam histórico precoce de delitos mais graves e repetitivos tendem a apresentar pontuações mais elevadas no PCL:YV; as pontuações do PCL:YV têm sido encontradas em associação com a presença de transtorno do comportamento disruptivo, alterações nas funções executivas e déficits nas relações interpessoais; em comparação com os meninos, os resultados do PCL:YV para as meninas não oferecem contribuições significativas quanto à reincidência criminal, violenta ou não.

$\mathrm{Na}$ realidade brasileira, estudos com instrumentos diagnósticos específicos para a psicopatia na adolescência são muito incipientes e os resultados ainda não generalizáveis, porém demonstram acompanhar as tendências internacionais na pesquisa de traços de psicopatia em jovens. Assim, o PCL:YV já está traduzido para a língua portuguesa do Brasil (Gauer, Vasconcellos, \& Werlang, 2006), bem como o seu manual, e sendo utilizado em pesquisas empíricas no meio acadêmico (Beheregaray, 2008; Ronchetti, 2009; Davoglio, 2009), especialmente voltadas para a busca de evidências de validade para o instrumento em amostras brasileiras (Davoglio, 2012; Ronchetti, Davoglio, Salvador-Silva, Vasconcellos \& Gauer, 2011).

As testagens psicológicas clássicas, por sua vez, utilizando informações de instrumentos como a Escala de Inteligência Wechsler para Crianças (WISC) ou a técnica de Rorschach, são abordagens que oferecem dados que confirmam os resultados encontrados nas escalas específicas para a avaliação da psicopatia. De acordo com Kernberg et al. (2003), quanto aos aspectos cognitivos, medidos pelo WISC, crianças e adolescentes com traços de psicopatia ou TPAS apresentam pontuações mais baixas na área verbal do que na de execução. Evidenciariam nisto suas limitações com o significado emocional das palavras, com a visão de conjunto, com a flexibilização de estratégias e com a percepção da conexão entre os elementos e as proporções. Já a técnica de Rorschach quando aplicado nessa população apresentou vazio de respostas de agressão, ausência de vínculos, alta taxa de egocentrismo, maior grau de grandiosidade e menor taxa de ansiedade (Kernberg et al., 2003). Esses elementos projetivos estariam revelando a desvalorização dos afetos alheios e a propensão à exploração emocional, características comumente encontradas na psicopatia.

A estabilidade dos comportamentos antissociais ao longo da adolescência ou nos períodos de transição entre as etapas de desenvolvimento foi identificada como uma preocupação recorrente nas pesquisas com adolescentes e crianças. Vuchinich, Bank e Paterson (1992) avaliaram 206 pré-adolescentes, por meio de um estudo longitudinal, iniciado quando os participantes tinham 10 e 12 anos. Os resultados indicaram que o comportamento antissocial permaneceu estável durante a pré-adolescência.

\section{Perspectivas atuais e futuras}

Com relação ao desenvolvimento de novas pesquisas com o construto da psicopatia aplicado à infância e à adolescência há ainda um grande número de questões fundamentais que se mantêm sem resposta, especialmente, quanto à utilidade do construto ou a melhor forma de conceituá-lo e medi-lo nestas populações. Diversos pesquisadores apontaram possibilidades futuras, a partir de suas próprias experiências empíricas. Selekin e Frick (2005), por exemplo, sugerem que as pesquisas deveriam focar na investigação da psicopatia levando em conta uma visão mais ampla das questões psicopatológicas da infância e adolescência, visando gerar novos conhecimentos sobre a etiologia, o grau de estabilidade temporal em relação ao desenvolvimento e o grau de comorbidades que deve ser esperado em amostras jovens. Para os autores, os estudos com essas amostras não deveriam restringir-se à busca de evidências apenas dos traços característicos da psicopatia adulta, estando abertas para considerar manifestações inusitadas em fases precoces.

Preocupações quanto à clara distinção entre o que é típico da adolescência e o que pode ser considerado expressão de psicopatia são referidas por vários autores (Seagrave \& Grisso, 2002; Skeem \& Cauffman, 2003), acirrando o debate sobre as implicações de estender o construto da psicopatia para os adolescentes. Eles argumentam que alguns aspectos da psicopatia (como por exemplo, irresponsabilidade, egocentrismo, falta de planejamento e impulsividade) são também características da juventude e, por isso, não podem ser incluídos como sinais/ sintomas de psicopatia. Entende-se, que tais argumentos, embora pertinentes, devam ser sempre relativizados a partir da história de vida de cada adolescente, levando-se em conta a intensidade, a frequência e as repercussões em diversos âmbitos de alguns aspectos que, a priori, acompanham a adolescência. É válido ressaltar, que a psicopatia deve ser enfocada como um construto dimensional, onde não se pressupõe uma avaliação categorial que considere a mera presença ou ausência de sinais/sintomas.

Há pesquisadores (Cicchetti \& Richters, 1997; Selekin \& Frick, 2005) que consideram essa dificuldade em distinguir normalidade e patologia como um risco inerente à pesquisa de quase todos os quadros psicopatológicos, partindo-se do princípio de que, frequentemente, os sintomas são uma intensificação ou variações de aspectos tidos como normativos. Desse modo, acredita-se que justamente a pesquisa empírica com populações jovens seja o caminho factível pode fornecer elementos elucidativos diante das preocupações referidas.

Com relação aos comportamentos delinquentes, as investigações atuais tendem a evidenciar que a idade de início e a persistência dos atos infracionais cometidos pelos adolescentes são importantes preditores da severidade e continuidade da prática de comportamentos antissociais (Farrington, 1995; Silva \& Rosseti-Ferreira, 2002; Tremblay, 2000). Ainda que os traços de psicopatia possam não ser absolutamente estáveis ao longo do desenvolvimento, especialmente na passagem da adolescência para a idade adulta, muitos pesquisadores acreditam que este pode ser um problema comum na avaliação de traços de personalidade. Para Selekin e Frick (2005) essa variabilidade na 
trajetória do transtorno psicopático ao longo do desenvolvimento, ao invés de um obstáculo, poderia ser útil para a investigação dos fatores que favorecem ou aumentam esta estabilidade, considerando também o reconhecimento dos fatores protetores para a criança ou adolescente.

Por outro lado, as comorbidades bastante comuns na infância e adolescência podem fazer com que os traços de psicopatia sejam confundidos com os encontrados em outros transtornos comuns a esta faixa etária, enfatizando preocupações quanto à diferenciação diagnóstica. Nesse sentido, os autores reafirmam que a essência da psicopatia manifesta-se nos aspectos afetivos e interpessoais, muito mais do que nos comportamentos transgressores (Frick, 2002; Kosson, Forth, Steuerwald, \& Kirkhart,1997). Deste modo, as pesquisas futuras poderiam explorar estratégias de avaliação por meio da combinação de diferentes informantes, além da própria criança, como professores, pais, pares, registros clínicos e institucionais (Farrington, 2005; Kamphaus \& Frick, 1996), priorizando as características afetivas e interpessoais dessas amostras.

Segundo Hare e Neumann (2008) novas investigações de traços psicopáticos em crianças e adolescentes são vitais para determinar suas trajetórias e sua adaptação e fixação (ou ausência delas) para outros indivíduos e para a comunidade em geral. Como apontam Kernberg e colaboradores (2003) seria muito mais produtivo se as pesquisas comparassem amostras de crianças ou adolescentes que apresentam traços do transtorno com aquelas que não apresentam, ao invés de compará-las com amostras adultas. Além disso, são recomendados esforços adicionais nas pesquisas com jovens no sentido de priorizar a compreensão dos fatores biológicos, neurológicos, psicológicos e sociais que dão início a personalidade psicopática e a mantém (Salekin, 2002).

Nesse sentido, Farrington (2005) apontou que os estudos longitudinais são essenciais, nos quais as medidas de traços de psicopatia devem ser repetidas em diferentes idades, embora reconheça ser um grande desafio a obtenção dessas amostras. $\mathrm{O}$ autor propôs até mesmo um roteiro sequencial de estudos envolvendo: (1) a definição e conceituação da Psicopatia adolescente, (2) a busca de evidências de validade de instrumentos diagnósticos, (3) os estudos longitudinais investigando fatores de desenvolvimento e risco, e (4) possíveis intervenções. Além disso, sugeriu que uma maior aplicação dos dados já conhecidos sobre a etiologia do transtorno, ainda que não sejam completos, possa servir de subsídio para o planejamento e desenvolvimento de programas preliminares de prevenção e tratamento.

\section{Considerações finais}

Os atuais resultados das pesquisas sobre traços de psicopatia na infância e adolescência, de acordo com literatura disponível, não são conclusivos, porém, evidenciam a prevalência dos problemas de externalização, de conduta ou antissociais nesta população. A presença dessas características em crianças e em adolescentes não determina, necessariamente, a atribuição de um diagnóstico clínico, ainda que, com frequência, essas características possam evoluir para quadros psicopatológicos, como TC, TPAS, psicopatia e comorbidades variadas.
Desse modo, diversos pesquisadores acreditam que seja bastante útil investigar os traços de psicopatia em adolescentes e crianças dentro da concepção geral de TP, considerando assim a sintomatologia associada aos aspectos desenvolvimentais, bem como a etiologia multifatorial. A possível presença de características afetivas e interpessoais da psicopatia (tais como a ausência de remorso, empatia ou sensibilidade afetiva) deveria ser apurada com meticulosidade, pois estas características constituem critérios de diferenciação diagnóstica de suma importância, se apresentadas concomitantemente aos aspectos comportamentais de natureza antissocial, estes por si não conclusivos para o diagnóstico de traços psicopáticos (Hare \& Neumann, 2008; Selekin \& Frick, 2005).

Observou-se também, por meio desta revisão da literatura, que, apesar dos atuais achados empíricos, a aplicação do construto da psicopatia à infância e à adolescência continua enfrentando divergências, especialmente, associadas a preocupações de ordem social e legal. Infere-se que algumas medidas propostas com o intuito de evitar rótulos e estigmatizações devido à atribuição desse diagnóstico, podem ser úteis em certa proporção, porém, trazem consigo, intrinsicamente, a relutância no reconhecimento e na abordagem objetiva desses sintomas, o que pode significar a inacessibilidade a qualquer tentativa terapêutica. Por outro lado, a cautela em oferecer diagnósticos psicopatológicos em crianças e adolescentes é bastante aceitável, porque, quando manejados inadequadamente poderiam, por exemplo, perpetuar a exclusão social

Contudo, pesquisadores como Kernberg et al. (2003) alertam que muitos prognósticos são reservados por si próprios, não tendo tanta relação com os riscos da nomeação diagnóstica, não devendo essa justificativa sobrepor-se ao necessário reconhecimento, visibilidade e atenção demandadas pelo sofrimento ou pela doença. Nesse sentido, é provável que quando oportuna e fundamentada, a identificação precoce de traços de psicopatia ofereça possibilidades de intervenções terapêuticas, psicossociais, educativas e inclusivas diferenciadas a esses jovens, aumentando as chances de uma melhor evolução do quadro apresentado. Acredita-se que diagnósticos precoces tendem sempre a ser de grande valor terapêutico, e isso tende a ser ainda mais verdadeiro quando se refere a quadros clínicos intimamente vinculados à construção e estruturação da personalidade. Nesse sentido, vale ressaltar que, por enquanto, as intervenções clínicas e psicossociais mais eficazes para o enfrentamento dos transtornos de personalidade continuam sendo medidas de promoção e prevenção da saúde mental, as quais corroboram a relevância da identificação de evidências diagnósticas incipientes.

Embora as pesquisas sobre psicopatia envolvendo crianças e adolescentes sejam árduas, demandem por cuidados éticos delicados que se ocupem de não estigmatizar ou segregar, e por ultrapassar barreiras clínicas, institucionais, legais e sociais, elas já avançaram consideravelmente nas duas últimas décadas em outros países. Mas para que a pesquisa científica com o construto, de fato, traga benefícios no diagnóstico e contribuições positivas, os estudos não podem prescindir de serem rigorosos, desde a qualificação e treinamento dos pesquisadores até a divulgação dos resultados fundamentada não na parcimônia, mas na 
transparência metodológica, que não dê margem a interpretações ambíguas, e na busca de evidências substanciais em diversos âmbitos da vida do examinando.

Ao chegar à realidade brasileira, a pesquisa sobre psicopatia em jovens carrega a vantagem de poder se apoiar nos avanços já obtidos por pesquisadores internacionais e em instrumentos psicométricos confiáveis, como o PCL:YV. É possível, no entanto, que os resultados dessas pesquisas, quer sejam em amostras brasileiras ou internacionais, encontrem seu mérito maior em beneficiar efetivamente as gerações futuras, representando, em última análise, um esforço visionário na tentativa de reduzir a violência e gerar qualidade de vida e de vínculos ao indivíduo e à sociedade.

\section{Referências}

Abrams, R. C., \& Horowitz, S. V. (1996). Personality disorders after age 50: A meta-analysis. Journal of Personality Disorders, 10, 271-281.

Achenbach, T. (1991). Manual for the child behavior checklis/4-18 profile. Burlington,VT: University of Vermont Department of Psychiatry.

American Academy of Child and Adolescent Psychiatry. (1997). Practice parameters for the assessment and treatment of children and adolescents with conduct disorder. Journal of the American Academy of Child \& Adolescent Psychiatry, 36(10), 122-139.

Associação Psiquiátrica Americana (2002). Manual Diagnóstico e Estatístico de Transtornos Mentais- Revisado (4 $4^{\mathrm{a}}$ ed). Porto Alegre: Artmed.

Beheregaray, A. P. (2008). Situação jurídico-penal e fatores de risco em adolescentes em conflito com a lei. (Dissertação de Mestrado). Pontifícia Universidade Católica do Rio Grande do Sul, Porto Alegre.

Brasil (1990). Estatuto da Criança e do Adolescente. Recuperado de http://www. planalto.gov.br/ccivil/Leis/L8069.htm

Calligaris, C. (2000). A adolescência. São Paulo: Publifolha.

Campbell, S. (1995). Behavioral problems in preschool children: A review of recent research. Journal of Child Psychology and Psychiatry, 36, 113-149.

Chandler, M., \& Moran, T. (1990). Psychopathy and moral development: A comparative study of delinquent and non-delinquent youth. Development and Psychopathology, 2, 227-246.

Cicchetti, D., \& Richters, J. E. (1997). Examining the conceptual and scientific underpinnings of research in developmental psychopathology. Development and Psychopathology, 9, 189-192.

Cleckley, H. (1988). The mask of sanity. St. Louis, MO: Mosby. (Texto original publicado em 1941).

Cooke, D. J., \& Michie, C. (2001). Refining the construct of psychopathy: towards a hierarchical model. Psychological Assessment, 13, 171-188.

Davoglio, T. R. (2009). Aspectos psicoafetivos e interações interpessoais em adolescentes em conflito com a lei. (Dissertação de Mestrado). Pontifícia Universidade Católica do Rio Grande do Sul, Porto Alegre.

Davoglio, T. R. (no prelo). Avaliação psicológica, psicopatologia e alteridade: Alguns caminhos epistemológicos. Psicologia:Ciência e Profissão, CFP.

Davoglio, T. R. (2012). Instrumentos de avaliação de traços da personalidade psicopática em jovens sul-brasileiros: Evidencias de validade. (Tese de Doutorado). Pontifícia Universidade Católica do Rio Grande do Sul, Porto Alegre.

Davoglio, T. R., \& Argimon, I. I. L. (2010). Avaliação de comportamentos antissociais e traços psicopatas em Psicologia Forense. Avaliação Psicológica, 9, 111-118.

Earls, F. (1994). Oppositional-defiant and conduct disorders. In M. Rutter, E. Taylor, L. A. Hersov (Orgs.), Child and adolescent psychiatry: Modern approaches (pp. 308-29). Oxford: Blackwell Scientific Publications.

Farrington, D. P. (1995). The challenge of teenage antisocial behavior. In M. Rutter
(Org.). Psychosocial disturbances in young people: Challenges for prevention (pp. 83-130). Cambridge: Press Syndicate of the University of Cambridge.

Farrington, D. P. (2005). The importance of child and adolescent psychopathy. Journal of Abnormal Child Psychology, 33(4), 489-497.

Fischer, M., Rolf, J., Hasazi, J. E., \& Cummings, L. (1984). Follow-up of a preschool epidemiological sample: Cross-age continuities and prediction of later adjustment with internalizing and externalizing dimensions of behavior. Child Development, 55, 137-150.

Forth, A. E., \& Burke, H. C. (1998). Psychopathy in adolescence: assessment, violence and developmental precursors. In D. J. Cooke, A. E. Forth \& R. D. Hare (Orgs.), Psichopathy: Theory, Research and Implications for Society (pp. 205-229). Dordrecht, The Netherlands: Kluwer.

Forth, A. E., Hart, S. D., \& Hare, R. D. (1990). Assessment of psychopathy in young male offenders. Psychological Assessment, 2, 342-344.

Forth, A. E., Kosson, D. S., \& Hare, R. D. (2003). The Psychopathy Checklist: Youth Version - Manual. Toronto, ON: Multi-Health Systems.

Frick, P. J. (2002). Juvenile psychopathy from a developmental perspective: Implications for construct development and use in forensic assessments. Law and Human Behavior, 26, 247-253.

Frick, P. J., \& Hare, R. D. (2001). Antisocial Process Screening Device. Toronto, ON: Multi-Health Systems.

Gauer, G. J. C., Vasconcellos, S. J. L., \& Werlang, B. G. (2006). Inventário de Psicopatia de Hare: Versão Jovens. Toronto, ON: Multi-Health Systems . Não publicado.

Hare, R. D. (2003). The Hare Psychopathy Checlist - Revised. Toronto, ON Multi-Health Systems.

Hare, R. D., \& Neumann, C. S. (2008). Psychopathy as a clinical and empirical construct. Annual Review of Clinical Psychology, 4, 217-46.

Johnson, J. G., McGeoch, P. G., Caskey, V. P., Abhary, S. G., Sneed, J. R., \& Bornstein, R. F. (2005). The developmental psychopathology of personality disorders. In B. L. Hankin \& J. R. Z. Abela (Orgs.), Development of psychopathology: A vulnerability-stress perspective (pp. 417-464). Thousand Oaks, California: Sage Publications.

Kamphaus, R. W., \& Frick, P. J. (1996). Clinical Assessment of Child and Adolescent Personality and Behavior. Boston: Allyn \& Bacon.

Keenan, K., \& Shaw, D. (1998). The development of coercive family processes: The interaction between aversive toddler behavior and parenting factors. In J. McCord (Org.), Coercion and punishment in long-term perspectives (pp. 165-180). Cambridge: Cambridge University Press.

Kernberg, P. F., Weiner, A. S., \& Bardenstein, K. K. (2003). Transtornos de Personalidade em Crianças e Adolescentes. Porto Alegre: Artmed.

Kosson, D. S.; Forth, A. E.; Steuerwald, B. L., \& Kirkhart, K. J. (1997). A new method for assessing behavior of psychopathic individuals: preliminary validation studies. Psychological Assesment, 9(2), 89-101.

Lambert, E., Wahler, R., Andrade, A., \& Bickman, L. (2001). Looking for the disorder in conduct disorder. Journal of Abnormal Psychology, 110(1), 110-123.

Loeber, R., Burke, J. D., Lahey, B. B., Winters, A., \& Zera, M. (2000). Oppositional defiant and conduct disorder: A review of the past 10 years, part I. Journal of the American Academy of Child \& Adolescent Psychiatry, 39, 1468-1484

Loeber, R., \& Dishion T. (1983). Early predictors of male delinquency: a review. Psychological Bulletin, 94, 68-99.

Lynam, D. R. (1997). Pursuing the psychopath: Capturing the psychopath in a nomological net. Journal of Abnormal Psychology, 106, 425-438.

Lynam, D. R. (1998). Early identification of the fledgling psychopath: Locating the psychopathic child in the current nomenclature. Journal of Abnormal Psychology, 107, 566-575.

Michaud, Y. (1989). A Violência. São Paulo: Ática.

Moffitt, T. (1993). Adolescence-limited and life-course-persistent antisocial behavior: A developmental taxonomy. Psychological Review, 100, 674-701.

Morana, H. (2004). Escala Hare PCL-R: Critérios para Pontuação de Psicopatia 
Revisados-Versão Brasileira. São Paulo: Casa do Psicólogo.

O’Connor, B. P., \& Dyce, J. A. (2001). Rigid and extreme: A geometric representation of personality disorders in five-factor model space. Journal of Personality and Social Psychology, 81, 1119-1130.

Organização Mundial da Saúde (2007). Classificação estatística internacional de doenças e problemas relacionados à saúde (CID-10). 10 a ed. Porto Alegre: Artes Médicas.

Organización Panamericana de la Salud. (1985). La salud del adolescente y el joven en las Américas / Health of adolescents and youth in the Américas. Washington, D.C. Publicación Científica.

Pacheco, J., Alvarenga, P., Reppold, C., Piccinini, C. A., \& Hutz, S. (2005). Estabilidade do comportamento anti-social na transição da infância para a adolescência: uma perspectiva desenvolvimentista. Psicologia: Reflexão e Crítica, 18(1), 55-61.

Patterson, G. R., DeGarmo, D. S., \& Knutson, N. (2000). Hyperactive and antisocial behaviors: Comorbid or two points in the same process? Development and Psychopathology, 12, 91-106.

Patterson, G., Reid, J., \& Dishion, T. (1992). Antisocial boys. Eugene: Castalia Pilotti, F. (1995). Crise e perspectivas da assistência à infância na América Latina. In F. Pilotti, \& I. Rizzini (Orgs.). A arte de governar crianças: A História das políticas sociais, da Legislação e da Assistência à Infância no Brasil (pp.10-45). Rio de Janeiro: Editora Universitária Santa Úrsula.

Pettit, G., Bates, J., Dodge, K., \& Meece, D. (1999). The impact of after-school peer contact on early adolescent externalizing problems is moderated by parental monitoring, perceived neighborhood safety, and prior adjustment. Child Development, 70, 768-778.

Robins, L. N. (1991). Conduct disorders. Journal Child Psychological Psychiatry, 32,193-212.

Ronchetti, R. (2009). Estudo de revisão e fidedignidade e consistência do Inventário de Psicopatia de Hare: Versão Jovens (PCL: YV). (Dissertação de Mestrado, Pontifícia Universidade Católica do Rio Grande do Sul, Porto Alegre).

Ronchetti, R., Davoglio, T. R., Salvador-Silva, R., Vasconcellos, S. J. L., \& Gauer, G.J.C. (2010). Inventário de Psicopatia de Hare: Versão Jovens (PCL:YV): Estudo preliminar em amostra adolescente brasileira Interamerican Journal of Psychology,44,536-542. Disponível em http://redalyc.uaemex.mx/src/ inicio/ArtPdfRed.jsp?iCve $=28420658017$

Ruegg, R., \& Frances, A. (1995). New research in personality disorders. Journal of Personality Disorders, 9, 1-48.

Rutter, M. (1992). Adolescence as a transition period: continuities and discontinuities in conduct disorder. Journal Adolescent Health, 13, 451-460.

Salekin, R. T. (2002). Psychopathy and therapeutic pessimism: clinical lore or clinical reality? Clinical Psychology Review, 22(1), 79-112.

Salekin, R. T., \& Frick, P.J. (2005). Psychopathy in children and adolescents: The need for a developmental perspective. Journal of Abnormal Child Psychology, 33(4), 403-409.

Seagrave, D., \& Grisso, T. (2002). Adolescent development and the measurement of juvenile psychopathy. Law and Human Behavior, 26, 219-239.

Skeem, J. L., \& Cauffman, E. (2003). Views of the downward extension: Comparing the youth version of the Psychopathy Checklist with the Youth Psychopathic Traits Inventory. Behavioral Sciences and the Law, 21, 737-770.

Silva, A. P., \& Rossetti-Ferreira, C. (2002). Continuidade/descontinuidade no envolvimento com o crime: Uma discussão crítica da literatura na psicologia do desenvolvimento. Psicologia: Reflexão e Crítica, 15(3), 573-585.

Tremblay, R. (2000). The development of aggressive behaviour during childhood: What have we learned in the past century? International Journal of Behavioural Development, 24(2), 129-141.

Trevothan, S. D., \& Walker, L. J. (1989). Hypothetical versus real life moral reasoning among psychopathic and delinquent youth. Development and Psychopathology, 1, 91-103.

UNICEF (2009). The state of the world's children 2009. Recuperado de http:// www.unicef.org/sowc09/docs/SOWC09-FullReport-EN.pdf

Veirmeiren, R. (2003). Psycopathology and delinquency in adolescents: A descriptive and developmental perspective. Clinical Psychology Review, 23, 277-318.

Vincent,G. M., Odgers, C. L., McCormick, A. V., \& Corrado, R. R. (2008). The PCL: YV and recidivism in male and female juveniles: A follow-up into young adulthood International Journal of Law and Psychiatry, 31, 287-296.

Vuchinich, S., Bank, L., \& Patterson, G. (1992). Parenting, peers and the stability of antisocial behavior in preadolescent boys. Developmental Psychology, $28,510-521$.

Wangby, M., Bergman,L., \& Magnusson, D. (1999). Development of adjustment problems in girls: what syndromes emergence? Child Development, 70(3) 678-699.

${ }^{1}$ Transtorno de personalidade antissocial (TPAS) é a classificação nosográfica atual que mais se aproxima e que veio a substituir no DSM o transtorno de personalidade psicopática, este não mais incluso nas últimas edições do manual, embora os especialistas não considerem os dois diagnósticos equivalentes (ver Ronchetti, 2009).

Tárcia Rita Davoglio, doutora em Psicologia Clínica pela Pontifícia Universidade Católica do Rio Grande do Sul, é pesquisadora e bolsista de Pós-Doutorado CAPES/FAPERGS na Pontifícia Universidade Católica do Rio Grande do Sul (PUCRS). Endereço para correspondência: Avenida Protásio Alves 2365, Sala 603, Bairro Petrópolis. CEP 90410-002. Porto Alegre, RS. E-mail: tarciad@gmail.com; tarcia.davoglio@pucrs.br Gabriel José Chittó Gauer, pós-Doutor em Psicologia pelo Departamento de Psicologia, Maryland University at College Park, é professor Titular na Pontifícia Universidade Católica do Rio Grande do Sul (PUCRS) e bolsista de produtividade do CNPq. E-mail: gabrielgauer@gmail.com

João Vitor Haeberle Jaeger, psicólogo graduado pela Pontifícia Universidade Católica do Rio Grande do Sul. E-mail: joaojaeger@hotmail.com

Marina Davoglio Tolotti, graduanda em Psicologia pela Pontifícia Universidade Católica do Rio Grande do Sul. E-mail: matolotti@hotmail.com 